\title{
A novel hybrid IWO and CS algorithm for phase- only pattern synthesis of large array antenna
}

\author{
Chao Liu*, Bo Yang, and Chuan Li \\ Department of Computer and Information Engineering, Hefei University of Technology, Hefei, \\ 230601, China
}

\begin{abstract}
In this paper, a novel hybrid Invasive Weed Optimization (IWO) and Cuckoo Search (CS) algorithm (IWO/CS) is presented for phase-only pattern synthesis of large array antenna. The hybrid IWO/CS algorithm embeds the Levy flight of CS algorithm into IWO algorithm as a global guide, thereby enhancing the global search ability of the algorithm. At the same time, in order to avoid trapping into the local optimal solution, mutation operator is introduced to enhance search ability of the algorithm. Finally, competitive exclusion operation is improved to maintain the diversity of population. The hybrid algorithm is applied to phase-only beam shaping to achieve main lobe broadening and side lobe depression. Simulation results demonstrate the superiority of the hybrid IWO and CS algorithm.
\end{abstract}

\section{Introduction}

In recent years, more and more researchers paid their attention to the field of array pattern synthesis as the core issue of antenna research. In the research of pattern synthesis, the main attention is to find the appropriate amplitude and phase of excitation to achieve the desired pattern. However, in many occasions, only phase shifters can be used of the beam control. And the beamforming algorithms based on amplitude and phase weighting are no longer applicable. Therefore, the research and implementation of phase-only pattern synthesis technology has very important theoretical and practical value.

At present, a lot of researches have been focused on the phase-only beamforming. Alexander [1] proposed a method to realize adaptive beam nulls through iterative minimization. Benjamin Fuchs [2] using the semi positive definite relaxation technique, converted the non-convex problem into a convex optimization solution, which was successfully applied to phase-only weighted beam control. In [3], phase-only weighted of low side lobe and pattern nulls were realized by using iterative phase perturbation technique. However, these methods are mainly applied to low side lobe and beam nulls, and the main lobe broadening ability is weak.

The intelligent optimization algorithm originated from biology has been widely concerned because of its strong search and global optimization ability, such as Particle Swarm Optimization [4], Backtracking Search Optimization [5], Cat Swarm Optimization

\footnotetext{
* Corresponding author: disneyl@hfut.edu.cn
} 
[6], and many others. They have been widely applied in the field of array signal processing. In [7], the IWO was applied to optimize the antenna problems, which was verified that the IWO is better than particle swarm optimization algorithm and genetic algorithm in convergence speed and stability. In [8], the CS algorithm was introduced to optimize the welded beam structures. Dhanesh [9] applied the differential evolution algorithm to the position-phase synthesis optimization problem. In [10], a hybrid IWO/WDO algorithm was proposed to implement pattern nulls by position-only control. However, there are still many problems to be improved in the phase-only beam shaping. At present, there are few methods to realize the main lobe widen by optimizing the phase when the amplitude is identical.

In this paper, a hybrid IWO/CS optimization algorithm is proposed for phase-only beam shaping of planar array. The hybrid algorithm introduces the Levy flight of the CS algorithm on the basis of the IWO algorithm as a global guide. Meanwhile, the mutation strategy is introduced to improve the local search performance of the algorithm. Finally, it is proposed to improve the competition exclusion mechanism, so that more seeds have the opportunity to reproduce. In order to achieve the beamforming requirements, the array antenna pattern is divided into three parts. Then, a different beamforming strategy is designed for each part in the fitness function. Compared with IWO/WDO algorithm, the performance of the hybrid IWO/CS algorithm is significantly improved, and it has a good performance in main lobe widen and side lobe level suppression.

\section{Hybrid IWO/CS algorithm}

The CS algorithm derived from cuckoo flight which has Levy flight properties. And it has good global search capabilities, but it is insufficient in local search capabilities. The IWO algorithm has good global and local search capabilities, but the convergence speed is slow and easy to trap in the local optimum when dealing with high-dimensional complex problems. In order to overcome the above shortcomings, Levy flight is introduced on the basis of IWO algorithm. Then, mutation operation is applied to the offspring to improve the local search ability. Finally, in order to maintain the diversity of population, a modified global random selection is applied to the competitive exclusion mechanism. The details are as follows.

1) Levy flight: Each seed in the population searches or explores globally according to Levy flight. The Levy flight update seed location formula is as follows:

$$
\mathbf{x}_{\text {temp }}=\mathbf{x}+\alpha \oplus \operatorname{Levy}(\lambda)
$$

where $\mathbf{x}$ and $\mathbf{x}_{\text {temp }}$ represent the current solution and new solution, respectively. $\alpha$ represents the step scale factor, the product $\oplus$ means entry-wise multiplication, $\operatorname{Levy}(\lambda)$ is Levy random search path, which subjects to the Levy distribution, it can be expressed as

$$
\text { Levy } \sim u=t^{-\lambda},(1<\lambda \leq 3)
$$

2) Greedy selection: The fitness value of the seed via Levy flight is calculated. If the fitness value of the current seed is better than that of the previous generation, the position of the seed is updated, otherwise the original position remain unchanged.

3) Reproduction: The seeds of weeds can reproduce asexually. The number of new seeds produced by each weed is determined by its fitness function.

$$
s=f l o o r\left[\frac{f-f_{\min }}{f_{\max }-f_{\min }}\left(s_{\max }-s_{\text {min }}\right)+s_{\text {min }}\right]
$$


where $f, f_{\max }$ and $f_{\min }$ denote current, maximum and minimum fitness value respectively, $s_{\max }$ and $s_{\min }$ are the maximum and minimum number of seeds, respectively.

4) Spatial dispersal: The seeds generated by asexual propagation are randomly distributed around the parent seeds in the normal distribution way. The standard deviation of normal distribution is defined as follows

$$
\sigma=\left(\frac{\text { iter }_{\max }-\text { iter }}{\text { iter }_{\max }}\right)^{n} \cdot\left(\sigma_{\text {initial }}-\sigma_{\text {final }}\right)+\sigma_{\text {final }}
$$

where iter denotes the number of iteration, $\sigma$ denotes standard deviation values, $n$ is the nonlinear factor.

The location of the new seed is defined as follows

$$
\mathbf{x}=\mathbf{x}_{\text {temp }}+\mathbf{r}_{1} \cdot \sigma
$$

where $\mathbf{r}_{1}$ is a random vector with uniform distribution in the range of [0,1].

5) Mutation: For each seed, two individuals are randomly selected from all the seeds, and the mutation operation is carried out for each dimension of the seed according to $r$. Mutation operations are defined as follows:

$$
\mathbf{x}_{\mathrm{i}}= \begin{cases}\mathbf{x}_{i}+\mathbf{r}_{2} \odot\left(\mathbf{x}_{j}-\mathbf{x}_{k}\right) & \mathbf{r}_{3}<r \\ \mathbf{x}_{i} & \mathbf{r}_{3}>=r\end{cases}
$$

where $\mathbf{r}_{2}$ and $\mathbf{r}_{3}$ are random vectors uniformly distribution in the range of [0,1]. $\mathbf{x}_{1}$ represents the $i$ th individual in the population, $j$ and $k$ are two randomly selected individuals in the population, and $i, j, k$ are different from each other, $r$ is the mutation threshold.

6) Modified Competitive exclusion: In order to avoid the increasing population, it is necessary to adopt a competition mechanism in the population to retain the useful information to the greatest extent. When the population number exceeds the maximum population number, all individuals are sorted according to their fitness values. The best 80 percent of individuals were selected from the population, and then 20 percent of individuals were randomly selected from the remaining individuals, otherwise repeat step 1 until the algorithm meets the stopping criterion.

\section{Phase only pattern synthesis based on the hybrid algorithm}

\subsection{Phase only beam shaping of planar array}

Suppose a planar array composed of $N$ elements, the elements in the planar array are isotropic sources. Therefore, the radiation pattern of the array can be expressed by the array factor. Now, the far-field pattern can be written as

$$
\begin{gathered}
E(\theta, \phi)=\sum_{n=1}^{N} g(\theta, \phi) w_{n}^{*} e^{j k\left(x_{n} \sin \theta \cos \phi+y_{n} \sin \theta \sin \phi\right)}=g(\theta, \phi) \mathbf{w}^{H} \mathbf{a}(\theta, \phi) \\
\mathbf{w}=\left[w_{1}, \ldots, w_{N}\right]^{T}
\end{gathered}
$$




$$
\mathbf{a}(\theta, \phi)=\left[\begin{array}{c}
e^{j k\left(x_{1} \sin \theta \cos \phi+y_{1} \sin \theta \sin \phi\right)} \\
\vdots \\
e^{j k\left(x_{N} \sin \theta \cos \phi+y_{N} \sin \theta \sin \phi\right)}
\end{array}\right]
$$

where $k=2 \pi / \lambda$ denotes the wave number, $\lambda$ is wavelength, $g(\theta, \phi)$ is the identical radiation pattern of array antennas, $w_{\mathrm{n}}$ is the excite of $n$th antenna element, $\left[x_{n}, y_{n}\right]$ denotes the position vector of $n$th element,$\{\bullet\}^{H}$ denotes complex conjugate transpose.

In the phase-only pattern synthesis, the complex weight vector $\mathbf{w}$ can be describe as $\mathbf{w}=|\mathbf{w}| e^{j \varphi}$. Where $|\mathbf{w}|$ is the amplitude of weight, it remains unchanged in the optimization, and $\varphi$ denotes the phase of weight. The radiation pattern can be rewritten as

$$
E(\theta, \phi)=\sum_{n=1}^{N} g(\theta, \phi)\left(\left|w_{n}\right| e^{j \varphi_{n}}\right)^{*} e^{j k\left(x_{n} \sin \theta \cos \phi+y_{n} \sin \theta \sin \phi\right)}=g(\theta, \phi)\left(|\mathbf{w}| e^{j \varphi}\right)^{H} \mathbf{a}(\theta, \phi)
$$

The essence of phase-only beamforming is to find a suitable set of phase vectors of array excitations to make the pattern meet the expectation. The optimal excitation phase vector can be obtained by minimizing the error between the actual pattern and the desired pattern. Assuming that the desired pattern is $E_{d}$, the optimization problem can be written as follows

$$
\min _{\varphi} \sum_{(\theta, \phi) \in \Phi}\left|g(\theta, \phi)\left(|\mathbf{w}| e^{j \varphi}\right)^{H} \mathbf{a}(\theta, \phi)-E_{d}(\theta, \phi)\right|^{2}
$$

where $\Phi$ represents the whole observation angle space.

\subsection{Fitness function of hybrid IWO/CS algorithm}

In this section, a new fitness function is proposed for main lobe broadening and side lobe depression. The design of the fitness function takes into account the desired optimization objectives and the actual ability of phase-only weighting. Therefore, the array pattern is divided into three parts: main lobe area, transition zone and side lobe area. The main lobe area is all considered in the fitness function. The side lobe area is only considered to be higher than the given side lobe level, and the transition band is not optimized to make the natural transition from the main lobe area to the side lobe area. The fitness function is formulated as follows

$$
f i t=\beta \sum_{(\theta, \phi) \in \Phi_{M}}\left[\left(|\mathbf{w}| \mathrm{e}^{j \varphi}\right)^{H} \mathbf{a}(\theta, \phi)\right]^{2}+\sum_{(\theta, \phi) \in \Phi_{S}}\left\{\max \left[\left(|\mathbf{w}| \mathrm{e}^{j \varphi}\right)^{H} \mathbf{a}(\theta, \phi)-S_{d B}, 0\right]\right\}^{2}
$$

where $S_{d B}$ is desired side lobe level, $\beta$ is the weight coefficient used to adjust the weight between the main lobe and the side lobe. $\Phi_{\mathrm{M}}$ and $\Phi_{\mathrm{S}}$ denote the main lobe area and side lobe area, respectively.

\subsection{Summarization of hybrid IWO/CS algorithm}

In the optimization problem of the phase-only array pattern, weeds are equivalent to feasible solutions for the excitation phase of the array. In conclusion, the basic steps of the IWO/CS algorithm can be summarized as follows 
Objective function fit $(\varphi), \boldsymbol{\varphi}=\left[\varphi_{1}, \ldots, \varphi_{N}\right]^{T}$

Initial a population of $\mathrm{m}$ weeds $\varphi_{i}(i=1,2, \ldots, m)$;

while (iter $<$ MaxIter) or (stop criterion)

Get a seed $\varphi$ by Levy flight in (1)

Calculate its fitness fit by (10), and update personal best phase by greedy selection

Calculate the number of next generation seeds per weed $\varphi$ by (3)

Calculate the standard deviation of normal distribution by (4), then the offspring $\varphi$ is obtained by (5)

The offspring $\varphi$ mutated through (6)

if $\left(P>P_{\max }\right)$

Update the population through the modified competitive exclusion

end

Keep the best solutions $\varphi_{i}$

end while

\section{Numerical results}

In this section, two kinds of planar arrays with different structures are simulated respectively, and the simulation results are compared with those in [10]. At the same time, the excitation amplitude of array element can be written as follows

$$
\left|w_{n}\right|=1, n=1,2 \ldots N
$$

In the first group of simulation experiments, a uniform planar array composed of $15 \times 15$ elements is considered. The array elements are isotropic and the spacing between the elements is half wavelength. The main lobe is widened in $\theta \in\left[0^{\circ}, 8^{\circ}\right]$ and $\phi \in\left[0^{\circ}, 360^{\circ}\right]$, and the side lobe level is reduced as much as possible in $\theta \in\left[12.5^{\circ}, 60^{\circ}\right]$ and $\phi \in\left[0^{\circ}, 360^{\circ}\right]$. The comparison results of the array patterns are shown in Table 1.

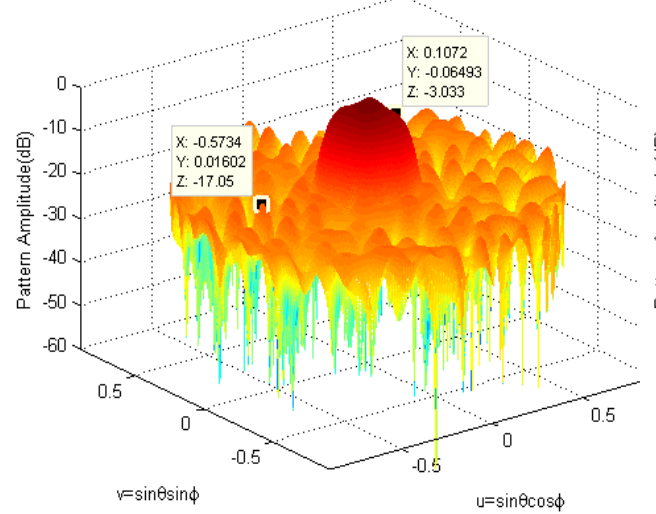

Fig. 1. Beam pattern of IWO/WDO

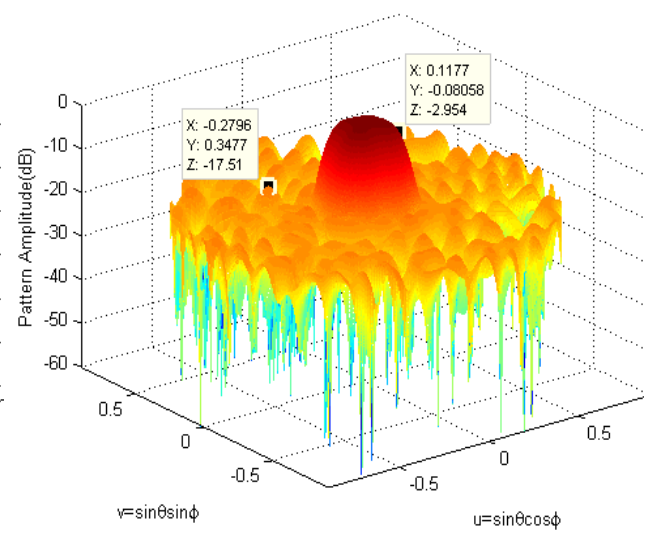

Fig. 2. Beam pattern of IWO/CS

Table 1. Results of Experiment 1.

\begin{tabular}{|c|c|c|}
\hline Algorithm & MSLL(dB) & HPBW $\left(^{\circ}\right)$ \\
\hline IWO/WDO & -17.0 & $12^{\circ}$ \\
\hline IWO/CS & -17.5 & $15^{\circ}$ \\
\hline
\end{tabular}




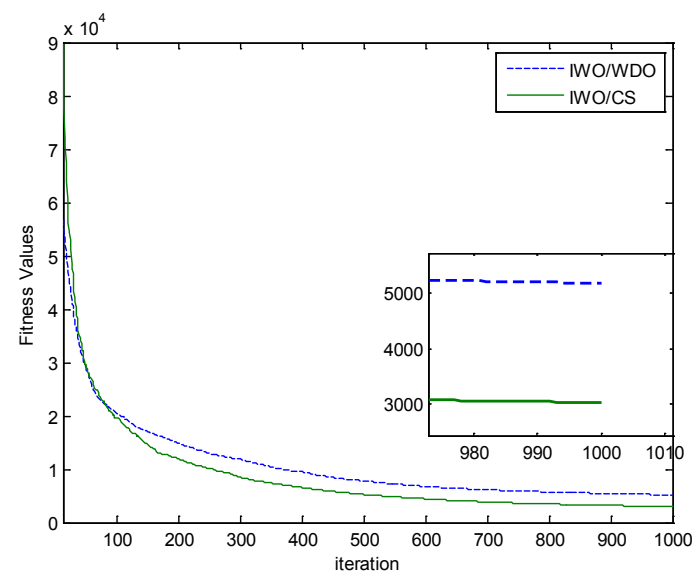

Fig. 3. Convergence curves of different methods.

From the above simulations, it can be seen that the maximum side lobe level of hybrid IWO/CS algorithm and comparison algorithm are $-17.5 \mathrm{db}$ and $-17 \mathrm{~dB}$, respectively. The $3 \mathrm{~dB}$ radius of main lobe is $7.5^{\circ}$ and $6^{\circ}$, respectively. Simultaneously, the convergence value of the fitness function is obviously better than that of the comparative algorithm.

In the second simulation experiments, a planar array composed of 664 elements is considered, which is shown in Fig.4. The array elements are isotropic and the spacing between elements is $0.8 \lambda$. The main lobe is widened in $\theta \in\left[0^{\circ}, 2.7^{\circ}\right]$ and $\phi \in\left[0^{\circ}, 360^{\circ}\right]$, and the side lobe level is reduced as much as possible in $\theta \in\left[5.5^{\circ}, 36^{\circ}\right]$ and $\phi \in\left[0^{\circ}, 360^{\circ}\right]$. Fig.5 and Fig.6 show the result of IWO/WDO algorithm and IWO/CS algorithm respectively, Fig. 7 shows the comparison of convergence curves of different algorithms, and the convergence value of IWO/CS is obviously better than that of the comparative algorithm. It can be seen that the maximum side lobe level of hybrid IWO/CS algorithm and comparison algorithm are $-18.9 \mathrm{~dB}$ and $-18.7 \mathrm{~dB}$, respectively. The main lobe width of the hybrid algorithm is $2.4^{\circ}$ better than the $2.2^{\circ}$ of the comparison algorithm. The comparison results of the array patterns are shown in Table 2.

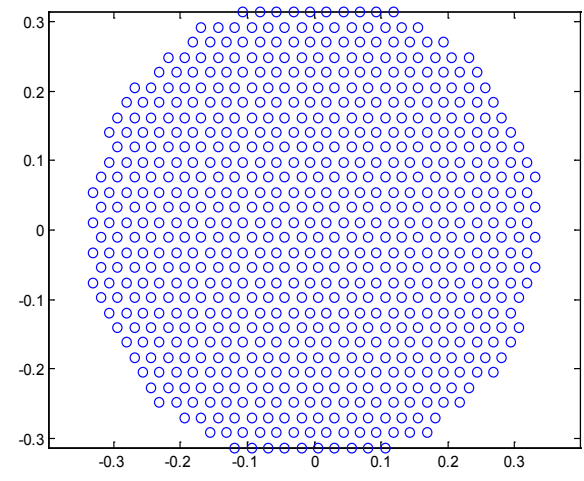

Fig. 4. 664 elements planar array

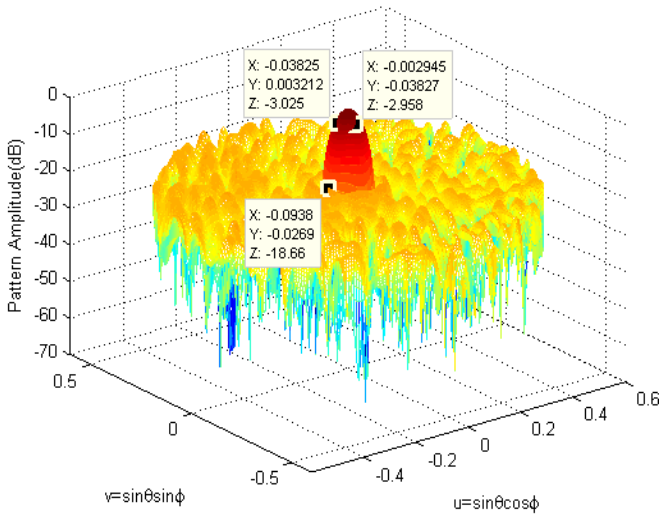

Fig. 5. Beam pattern of IWO/WDO 

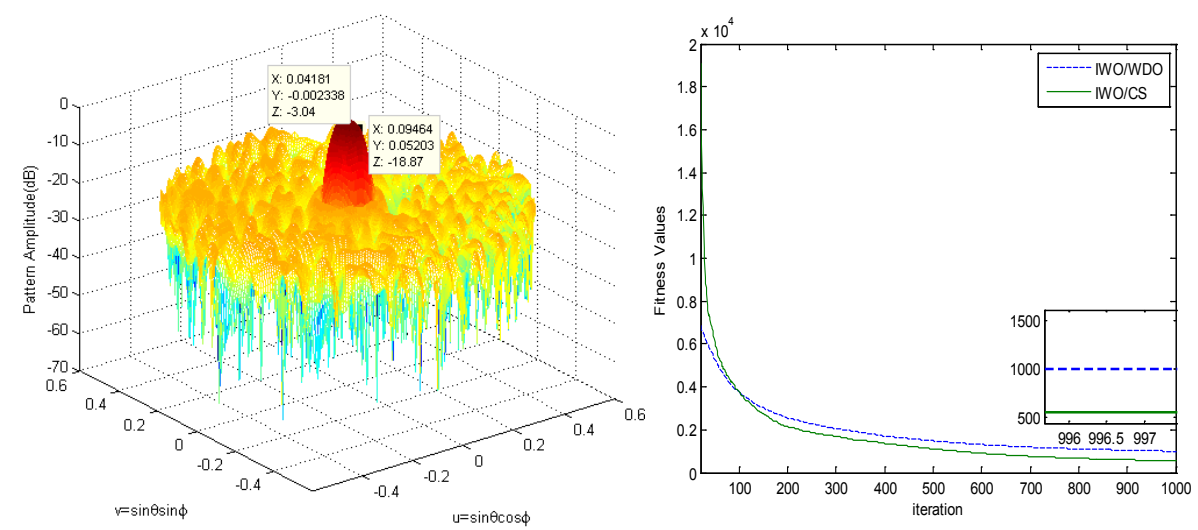

Fig. 6. Beam pattern of IWO/CS

Fig. 7. Convergence curves of different methods

Table 2. Results of Experiment 2.

\begin{tabular}{|c|c|c|}
\hline Algorithm & MSLL(dB) & HPBW( $\left(^{\circ}\right)$ \\
\hline IWO/WDO & -18.7 & $4.4^{\circ}$ \\
\hline IWO/CS & -18.9 & $4.8^{\circ}$ \\
\hline
\end{tabular}

From the above comparative experiments, it can be seen that the convergence performance of the fitness function of the hybrid IWO/CS algorithm is better than that of the IWO/WDO algorithm, and the main lobe width and side lobe level are better than the comparison algorithm at the same time. To sum up, the comprehensive effect of the hybrid algorithm presented in this paper is better than the IWO/WDO algorithm. The phase only shaping of planar array with arbitrary structure can be achieved.

\section{Conclusions}

In this paper, a novel hybrid IWO/CS algorithm is presented, which is applied to the phaseonly array pattern synthesis. In order to adapt to the characteristics of phase-only beamforming, the Levy flight is introduced on the basis of IWO algorithm to improve the ability of global optimization. To effectively avoid the local optimum, the mutation operation is performed on the offspring seeds. In addition, an improved competitive exclusion mechanism is applied to maintain the diversity of the population. Finally, according to the requirements of the desired pattern, a new fitness function is designed. Compare to IWO/WDO algorithm, the improved algorithm has superiority in convergence accuracy, side lobe level and main lobe width. Several simulation examples of different arrays demonstrate the effectiveness and robustness of the proposed algorithm.

\section{References}

1. A. D. Khzmalyan and A. S. Kondratiev, "The phase-only shaping and adaptive nulling of an amplitude pattern," IEEE Transactions on Antennas and Propagation, vol. 51, no. 2, pp. 264-272, Feb. (2003).

2. B. Fuchs, "Application of Convex Relaxation to Array Synthesis Problems," IEEE Transactions on Antennas and Propagation, vol. 62, no. 2, pp. 634-640, Feb. (2014). 
3. Y. Aslan, J. Puskely, A. Roederer and A. Yarovoy, "Phase-Only Control of Peak Sidelobe Level and Pattern Nulls Using Iterative Phase Perturbations, ” IEEE Antennas and Wireless Propagation Letters, vol. 18, no. 10, pp. 2081-2085, Oct. (2019).

4. M. M. Khodier and C. G. Christodoulou, "Linear array geometry synthesis with minimum sidelobe level and null control using particle swarm optimization, " IEEE Transactions on Antennas and Propagation, vol. 53, no. 8, pp. 2674-2679, (2005).

5. K. Guney, A. Durmus, and S. Basbug, "Backtracking search optimization algorithm for synthesis of concentric circular antenna arrays," International Journal of Antennas and Propagation, vol. 2014, Article ID 250841, 11 pages, (2014).

6. L. Pappula and D. Ghosh, "Linear antenna array synthesis using cat swarm optimization," AEU-International Journal of Electronics and Communications, vol. 68, no. 6, pp. 540-549, (2014).

7. S. Karimkashi and A. A. Kishk, "Invasive Weed Optimization and its Features in Electromagnetics," IEEE Transactions on Antennas and Propagation, vol. 58, no. 4, pp. 1269-1278, April (2010).

8. X. S. Yang and S. Deb, "Engineering Optimisation by Cuckoo Search." International Journal of Mathematical Modelling \& Numerical Optimisation, Vol. 1, No.4, 330-343 (2010).

9. D. G. Kurup, M. Himdi and A. Rydberg, "Synthesis of uniform amplitude unequally spaced antenna arrays using the differential evolution algorithm," IEEE Transactions on Antennas and Propagation, vol. 51, no. 9, pp. 2210-2217, Sept. (2003).

10. S. K. Mahto and A. Choubey, "A Novel Hybrid IWO/WDO Algorithm for Interference Minimization of Uniformly Excited Linear Sparse Array by Position-Only Control," IEEE Antennas and Wireless Propagation Letters, vol. 15, pp. 250-254, (2016) 